\title{
Robotic vesico-vaginal fistula repair with no omental flap interposition
}

\author{
Alberto Martini ${ }^{1}\left(\mathbb{D} \cdot\right.$ Eugenio Dattolo $^{1} \cdot$ Jacopo Frizzi $^{1}$ \\ Donata Villari ${ }^{1}$ - Maria Cristina Paoletti ${ }^{1}$
}

Received: 17 December 2015 / Accepted: 16 February 2016

(C) The International Urogynecological Association 2016

\begin{abstract}
Introduction and hypothesis A vesico-vaginal fistula (VVF) is a fistulous tract that connects bladder and vagina, causing urine leakage via the vagina. In the developed world, iatrogenic postoperative VVF is the most common case. Classically, when treating a VVF via the abdominal route, an abdominal flap is mobilized and interposed between the bladder and the vagina.

Methods In our video, we describe a robotic VVF repair technique with no omental flap interpositioning for a vaginal vault-located fistula.

Results Duration of surgery was 95 min, estimated blood loss was $<50 \mathrm{ml}$. The postoperative course was uneventful. At the 6-month follow-up, which included clinical and cystographic examinations, the patient had not experienced any recurrence. Conclusion In our opinion, a two-layered suturing technique using two semi-continuous sutures for vaginal closure and perpendicular interrupted stitches for bladder closure does not require omental flap mobilization, reducing operating time and possible complications related to accidental peritoneal injuries.
\end{abstract}

Electronic supplementary material The online version of this article (doi:10.1007/s00192-016-2989-5) contains supplementary material. This video is also available to watch on http://link.springer.com/. Please search for this article by the article title or DOI number, and on the article page click on 'Supplementary Material'

Alberto Martini

a.martini.md@gmail.com

1 Department of Urology, University of Florence, Careggi Hospital, Viale San Luca, 50134 Florence, Italy
Keywords Vesico-vaginal fistula $\cdot$ Robotic $\cdot$ Minimally invasive repair

\section{Aim of the video \\ To present and describe our minimally-invasive robotic transabdominal extravesical approach for treating a vesico- vaginal fistula (VVF) with no omental flap interposition.}

\section{Materials and methods}

We present the case of a 43-year-old patient with a VVF occurring 2 months after total abdominal hysterectomy including salpingo-oophorectomy for uterine fibromatosis. The patient's BMI was $24.09 \mathrm{~kg} / \mathrm{m}^{2}$. She presented with vaginal urinary leakage. Preoperative cystography helped us to identify the fistula located at the vaginal vault, distant from ureteral ostia.

Given the fistula's location and the patient's BMI, a minimally invasive robotic approach was chosen. We opted for this approach over open surgery because of its lower morbidity and associated quicker recovery. Over laparoscopy, the robotic approach allows for better visibility and dexterity.

Before the procedure, the patient was placed in the lithotomy position. The patient table was tilted at approximately $30^{\circ}$ to facilitate first cystoscopy and then bladder catheter positioning. Prophylactic intravenous antibiotics were administered before surgery.

The fistula was visualized under cystoscopy; a 5-Fr catheter was placed within the fistula and exited through the vagina. Ureteral ostia were located and two 5-Fr ureteral catheters were placed within the ureters. A 18-Fr Foley bladder catheter was placed intravesically. 
Adhesions were expected because of previous open surgery; thus, the first trocar $(12 \mathrm{~mm})$ was positioned according to the Hasson technique, $3 \mathrm{~cm}$ above the umbilicus.

The remaining four trocars were placed in a dome fashion under direct vision: an 8-mm port on the left and three ports on the right, one 8-mm and two 12-mm ports for laparoscopic assistance. The da Vinci ${ }^{\circledR}$ robot was placed between the patient's legs for docking.

Side docking has been described for gynecological robotic surgery. It offers ample space for two assistants, and better vaginal access [1]. Given that our technique accounts for one assistant laparoscopic surgeon only, and, except for initial valve positioning, it does not require transvaginal access, we adopted classical docking rather than side docking. Furthermore, as we used two robotic arms, this configuration allows symmetrical trocar positioning, thus minimizing potential intraoperative arm collisions.

Finally, the robotic arms were introduced; we used a ProGrasp ${ }^{\mathrm{TM}}$ forceps, Maryland bipolar forceps, monopolar curved scissors, and two large needle drivers. One assistant surgeon stood on the patient's right side to assist the console surgeon laparoscopically.

A vaginal valve, positioned into the anterior vaginal fornix, helped us to identify the anatomical dissection plan between the vagina and the bladder, along with the inflated urinary catheter's balloon, as demonstrated in the video.

After initial adhesiolysis, we performed sharp and blunt dissection using ProGrasp ${ }^{\mathrm{TM}}$ forceps first, then bipolar Maryland forceps on the left arm, and monopolar curved scissors on the right to access the vesico-vaginal space. After obtaining good exposure, we located the fistula.

The Foley catheter balloon was deflated and the catheter was withdrawn. The fibrotic margins of the fistulous tract were carefully resected. Extravesical repair was performed with no omental flap interposition. A two-layer closure technique was performed using 3-0 Monosyn ${ }^{\circledR}$. The vagina was sutured with two semi-continuous sutures in a horizontal fashion, starting at the opposite sides and then tied together on the midline.

Bladder closure was realized with interrupted stitches, placed perpendicular to the vaginal sutures. Peritoneal closure with interrupted stitches completed the operation. After a leakage test of the bladder, we removed all the ports.

\section{Results}

Docking time was $10 \mathrm{~min}$. Duration of surgery was $95 \mathrm{~min}$, with estimated blood loss of $<50 \mathrm{ml}$. The postoperative course was uneventful. On postoperative day 1 the patient reported pain of 3 out of 10 on a visual analog scale $(0=$ no pain; $10=$ unbearable pain). On postoperative day 2 , drainage was removed; the patient was discharged on day 4 with an $18-\mathrm{Fr}$ bladder catheter in situ. Twenty days later, after cystography, the urinary catheter was removed. At the 6-month follow-up the patient had not experienced any recurrence according to clinical and cystographic examinations.

\section{Conclusion}

For treating VVFs located above the bladder trigone a transabdominal approach is preferred rather than a transvaginal one. Either laparoscopic or robotic approaches have been described [2]. Surgeons can choose between the traditional transvesical (O’Connor repair) and the extravesical technique [2,3]. Classically, an omental flap is mobilized and interposed between the bladder and the vagina $[4,5]$. Omental interpositioning has never been proven to result in higher cure rates for VVF repair; nevertheless, few authors report no flap mobilization [2,3]. Miklos et al. claim a success rate of $98 \%$ without flap interposition over 15 years [2].

Robot-assisted VVF repair is a safe and feasible procedure. After 6 months the patient had not experienced any clinical or cystographic relapse. Thanks to the minimally invasive approach, blood loss is negligible.

In our opinion, a two-layered suturing technique using two semi-continuous sutures for vaginal closure and perpendicular interrupted stitches for bladder closure does not require omental flap mobilization, reducing operating time and possible complications related to accidental peritoneal injuries.

\section{Acknowledgements None.}

\section{Compliance with ethical standards}

\section{Conflicts of interest None.}

Consent Written informed consent was obtained from the patient for publication of this video article and any accompanying images.

\section{References}

1. Einarsson JI, Hibner M, Advincula AP (2011) Side docking: an alternative docking method for gynecologic robotic surgery. Rev Obstet Gynecol 4:123-125

2. Miklos JR, Moore RD, Chinthakanan O (2015) Laparoscopic and robotic-assisted vesicovaginal fistula repair: a systematic review of the literature. J Minim Invasive Gynecol 22:727-736

3. Miklos JR, Moore RD (2015) Laparoscopic extravesical vesicovaginal fistula repair: our technique and 15 -years experience. Int Urogynecol J 26:441-446

4. Dutto L, O'Reilly B (2013) Robotic repair of vesico-vaginal fistula with perisigmoid fat flap interposition: state of the art for a challenging case? Int Urogynecol J 24:2029-2030

5. Ezzat M, Ezzat MM, Tran VQ, Aboseif SR (2009) Repair of giant vesicovaginal fistulas. J Urol 181:1184-1188 